\title{
Downregulation of OCT4 promotes differentiation and inhibits growth of BE (2)-C human neuroblastoma I-type cells
}

\author{
LIN YANG ${ }^{*}$, JICUI ZHENG ${ }^{*}$, TING XU and XIANMIN XIAO \\ Department of Surgery, Children's Hospital of Fudan University, Shanghai 201102, P.R. China
}

Received October 19, 2012; Accepted January 11, 2013

DOI: $10.3892 /$ or.2013.2356

\begin{abstract}
In in vitro continuous culture, N (neuroblastic)-, $\mathrm{S}$ (substrate adherent)- and I (intermediate)-type cells were identified in human neuroblastoma (NB), where I-type is recognized as a stem cell type. Octamer-binding protein 4 (OCT4) is a cell marker used to identify the stemness of cells, whose roles in regulating I-type NB cells have yet to be fully elucidated. In the present study, we assessed the differentiation regulation role of OCT4 in BE (2)-C of I-type cells. We demonstrated that downregulation of OCT4 expression in $\mathrm{BE}$ (2)-C was associated with reduced cell proliferation and loss of colony formation ability on soft agar, and prompted BE (2)-C cells to differentiate to S-type cells. By contrast, overexpression of OCT4 increased cell proliferation and colony formation ratio, but no obvious differentiation promotion was observed. Furthermore, induced differentiation of BE (2)-C cells to S-type by 5-bromo-2'-deoxyuridine (BrdUrd) was accompanied by reduced expression of OCT4.
\end{abstract}

\section{Introduction}

Neuroblastoma (NB) is the most common malignant extra cranial solid tumor in children. NB is an extremely heterogeneous disease, both clinically and biologically, resulting from the plasticity of the embryonic neural crest, in which this tumor originates $(1,2)$. Clinically, it has been shown that cellular heterogeneity and extent of maturation (such as, stroma-rich and -poor tumors or high-and low-risk tumors, based on histological grade) correlate with clinical manifestation, and these properties have been used for the classification and prognosis of the disease (3). Cell lines established from human NB also show the same cellular heterogeneity. Based on morphological appearance, biochemical properties, and

Correspondence to: Dr Xianmin Xiao, Department of Surgery, Children's Hospital of Fudan University, 399 Wanyuan Road, Shanghai 201102, P.R. China

E-mail:xmxiao@shmu.edu.cn

*Contributed equally

Key words: octamer-binding protein 4, neuroblastoma, differentiation growth patterns, three major cell types have been identified in NB cell lines: N (neuroblastic)-, S (substrate adherent)- and I (intermediate)-type NB cells. Significant similarities between normal neural crest stem cells and I-type NB cells in selfrenewal, multipotent and differentiation ability may indicate that I-type cells represent a population of NB stem cells or malignant neural crest stem cells (4-6).

The octamer-binding protein 4 (OCT4), a member of the POU family of transcription factors, together with SOX-2 and NANOG, regulates self-renewal and differentiation in embryonic stem cells (ESCs) (7). Recent findings demonstrated that OCT4 also plays an oncogenic role and is expressed in some cancer stem cells (8-11). Furthermore, previous studies detected the expression of OCT4 in NB primary samples, metastatic bone marrow aspirates, cell lines, and OCT4 is facilitated to identify NB cancer stem cells as the stemness gene (12-17). OCT4 is proved to have a potential role in maintaining NB cancer stem cell niche localized in the hypoxic zones of the solid tumors in vivo (12) and is used for identification of tumor-derived endothelial cells as a putative marker (17).

In this study, we investigated OCT4 expression in the BE (2)-C human NB I-type cell line, typical NB cell line, and analyzed the possible relationship between the expression levels of OCT4 and tumor genesis. Our study demonstrates a role of OCT4 expression levels in the regulation of BE (2)-C self-renewal capacity and differentiation.

\section{Materials and methods}

Cell culture and differentiation assays. The BE (2)-C human NB I-type cell lines (ATCC CRL-2268) were cultured in DMEM with F12 (Sigma) containing 10\% fetal bovine serum (FBS; Invitrogen). In differentiation assays, all-trans retinoic acid (RA) and 5-bromo-2'-deoxyuridine (BrdUrd) were dissolved in DMSO and $10 \mathrm{mM}$ solutions were stocked. BE (2)-C cells were grown for two weeks in the presence of $10 \mu \mathrm{M}$ of RA or $10 \mu \mathrm{M}$ BrdUrd.

Lentiviral constructs and transfection. FUGW-OCT4 was constructed by replacing GFP with OCT4 ORF (NM_002701) of lentiviral vector FUGW (18). The sequence corresponding to OCT4 full length ORF was amplified from hESC cDNA library (Invitrogen, A10303-01) with AgeI and EcoRI restriction site added to $5^{\prime}$ and $3^{\prime}$ of the PCR fragment, respectively. The fragment was digested and ligated to FUGW vector that 
A

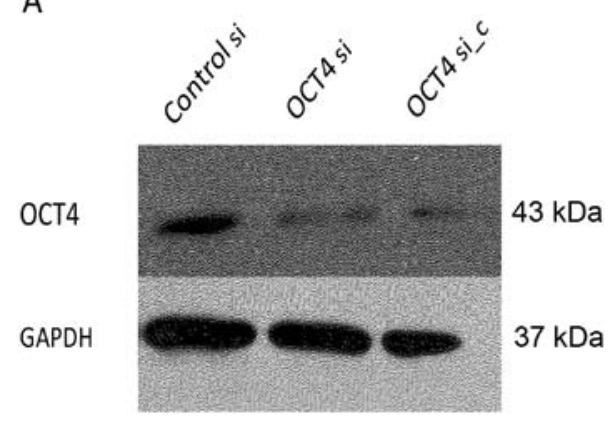

B

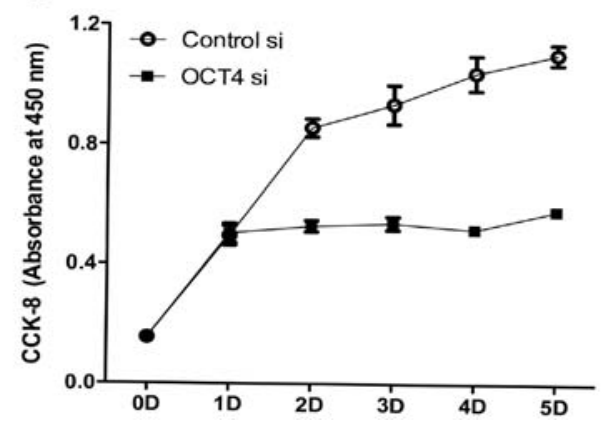

Figure 1. Downregulation of OCT4 in BE (2)-C cells. (A) Western blot analysis of OCT4 downregulation in BE (2)-c cells by siRNA. GAPDH levels are shown as control. (B) OCT4 downregulation inhibited proliferation of BE (2)-C cells. BE (2)-C cells transfected with OCT4 siRNA (OCT4 si) or non-functional siRNA (control si) were plated in 96-well plates. Cell growth rates were measured by Cell Counting Kit-8 (CCK-8) assay. Data are expressed as the means \pm SD with each point performed in triplicate. Independent experiments were repeated three times.

has a ubiquitin- $\mathrm{C}$ promoter used to overexpress human OCT4 in BE (2)-C cells.

To downregulate OCT4 expression, we constructed siRNA clones as following: oligonucleotides encoding shRNA were inserted downstream of and driven to express by a human U6 promoter. Each oligonucleotide pair contained a 5' AgeI and 3' EcoRI overhang; an RNA polymerase III termination sequence, the OCT4 target sequence and its antisense were separated by a piece of short loop sequence. For sequence OCT4 si_c CCCTCACTTCACTGCACTGTA, which was reported to be able to efficiently knock down OCT4, the loop is CTCGAG (19). Another target sequence was selected using BLOCK-iTTM RNAI Designer (Invitrogen). OCT4 si: CCGTGAAGCTGGAGAAGGA, while in this case TTCAAGAGA is the loop. A non-functional sequence TTCTCCGAACGTGTCACGT was included as control. Oligonucleotides were synthesized (Invitrogen), annealed, and ligated into the vector PSCN according to a previously described manipulation procedure (20).

Cell proliferation analysis. Cells were seeded onto 96-well plates at 2,500 cells/well and transfected with lentiviral vector after $12 \mathrm{~h}$. After culturing for various durations, cell growth rates were measured by using Cell Counting Kit-8 (CCK-8; Dojindo Laboratories), according the manufacturer's instructions.

Soft agar colony formation assay. After $24 \mathrm{~h}$ of transfection, cells were dissociated into single cell suspensions and mixed in $0.3 \%$ Noble agar (in DMEM containing 10\% FBS) and seeded onto 6 -well plates containing $0.6 \%$ Noble agar in the same growth medium at 1,000 cells/well. After 14 days of incubation, colonies were stained with $5 \mathrm{mg} / \mathrm{ml} \mathrm{MTT}$ and photographed. All experiments were carried out in triplicate.

Western blot analysis. Cells were lysed in 1\% Triton X-100 buffer. Total cell protein homogenates $(20 \mu \mathrm{g})$ were separated on SDS-polyacrylamide gels, transferred to a polyvinylidene fluoride membrane and probed with 1:1000 rabbit polyclonal Oct4 (Abcam), 1:5000 mouse monoclonal to $68 \mathrm{kDa}$ Neurofilament (Abcam), 1:1000 monoclonal anti-peripherin (mouse IgG1 isotype, Sigma), 1:1000 vimentin (R28) (Cell Signaling Technology), 1:1000 S100 $\alpha$ chain (Santa Cruz
Biotechnology, Inc.). Horseradish peroxidase-conjugated goat anti-rabbit and goat anti-mouse IgG were used as secondary antibodies. Mouse monoclonal anti-human GAPDH was used as internal control. The membranes were developed with a SuperSignal West Pico chemiluminescence kit (Pierce).

RNA extraction andreal-time quantitative reverse-transcription polymerase chain reaction (RT-PCR). Total RNA was extracted from cultured cells using TRIzol reagent (Invitrogen) and reverse transcribed into cDNA with the MMLV Reverse Transcriptase kit (Promega) according to the manufacturer's instructions. Quantitative RT-PCR analysis was carried out with the SYBR Premix Ex Taq (Takara, Dalian) using the Bio-Rad iQ5 realtime PCR system according to the manufacturer's instructions. Sequences for mRNAs from the nucleotide data bank (National Center for Biotechnology Information, USA) were used to design primer pairs with the PrimerBank website. Sequences were: human Oct4 (sense: GGGAGATTGATAACTGGTGTGTT, antisense: GTGTATATCCCAGGGTGATCCTC); human peripherin (sense: CCAAGTACGCGGACCTGTC, antisense: CTCGCACGTTAGACTCTGGA); human vimentin (sense: GAACGCCAGATGCGTGAAATG, antisense: CCA GAGGGAGTGAATCCAGATTA); human neurofilament 68 (NF68) (sense: ATGAGTTCCTTCAGCTACGAGC, antisense: GGGCATCAACGATCCAGAGC); human S100 (sense: GACCCTCATCAACGTGTTCCA, antisense: CCACAAGC ACCACATACTCCT). Appropriate regions of human $\beta$-actin were used as controls.

\section{Results}

Downregulation of OCT4 in BE (2)-C cells inhibits proliferation of BE (2)-C cells and promotes cell differentiation into $S$-type. Lentiviral vector produced from either constructs was effective in downregulating OCT4 expression in BE (2)-C cells, and $>90 \%$ of OCT4 protein was suppressed by RNA interference, insistent with si_c from other researcher's interference sequence (Fig. 1A). This effect was not observed in $\mathrm{BE}$ (2)-C cells infected with the control vector. A reduction in cell growth rate was observed in cells with downregulated OCT4 than the controls measured by CCK-8 absorption (Fig. 1B). 


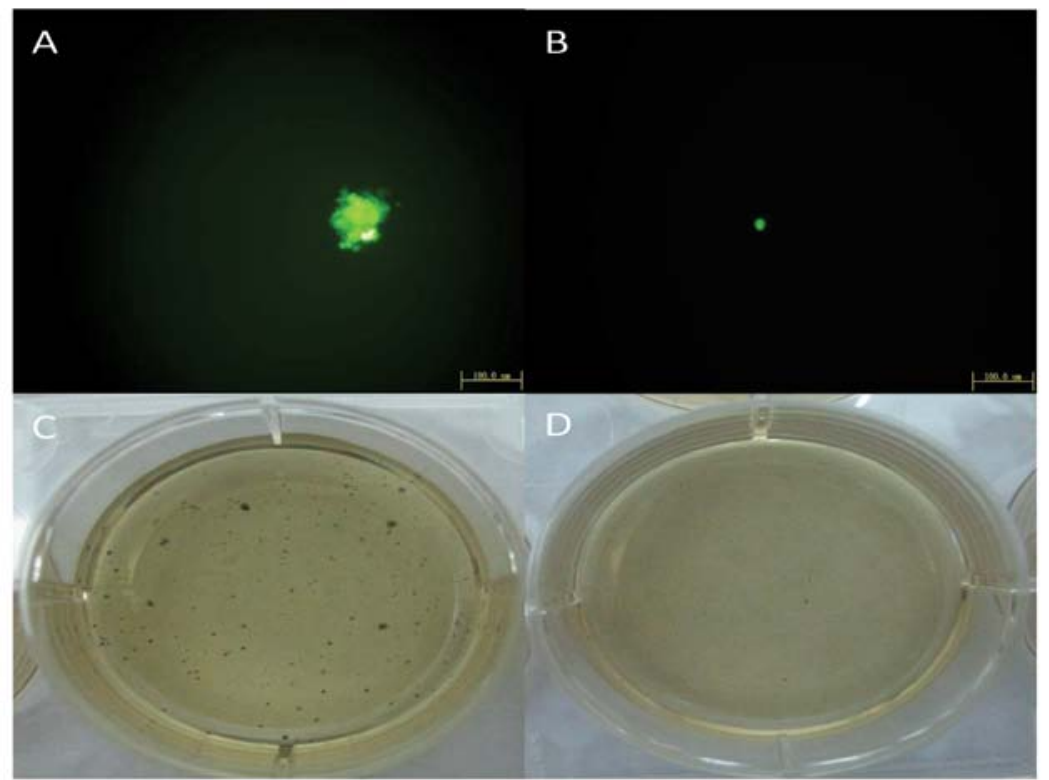

Figure 2. Measurement of self-renewal capacity of BE (2)-C cells with OCT4 downregulation using soft agar assay. (A) Micrographs of colonies formed from cells with empty vector (control si) in soft agar 14 days after plating. (B) Colonies formed from cells with OCT4 RNAi vector (OCT4 si). (C and D) Colonies in one well corresponding to (A and B), respectively; downregulation of OCT4 abrogated the ability of BE (2)-C cells to grow in soft agar. Cells $(1,000)$ were plated in each well. Viable colonies on soft agar were stained with $5 \mathrm{mg} / \mathrm{ml}$ MTT 14 days after plating. Colonies formed from cells with scale bar, $100 \mu \mathrm{m}$.
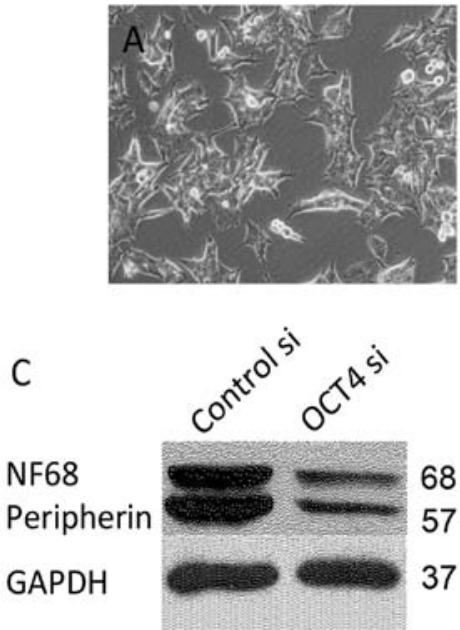

$\mathrm{kDa}$

$57 \mathrm{kDa}$

$37 \mathrm{kDa}$

E

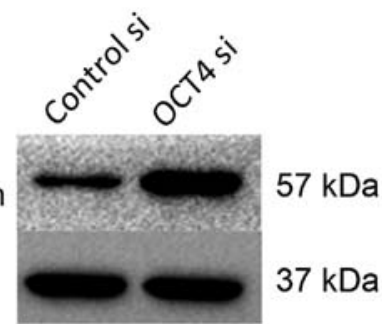

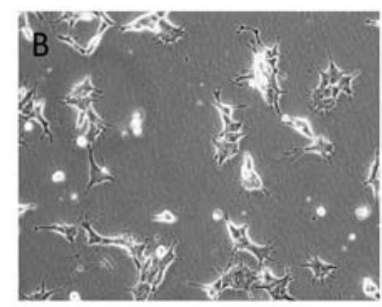

D
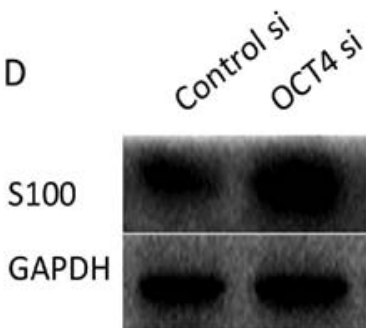

$11 \mathrm{kDa}$

$37 \mathrm{kDa}$

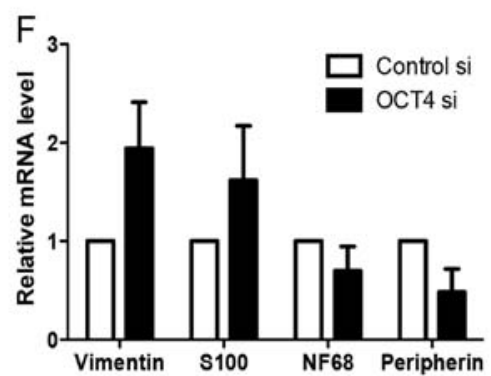

Figure 3. Differentiation of OCT4 downregulated cells. (A) BE (2)-C/control si and (B) BE (2)-C/OCT4 si morphology. BE (2)-C/OCT4 si cells adhered tightly to the underlying substrate and formed a relatively contact-inhibited monolayer. (C) N-type markers of NF68 and peripherin. (D) S-type marker of S100. (E) S-type marker of vimentin. (F) Quantitative RT-PCR of 4 lineage markers indicating relative expression levels. All results were from cells transfected after 14 days.

OCT4 si cells showed morphological alteration of $\mathrm{S}$ type cells (Fig. 3B) and the soft agar assay indicated colony formation of cells with downregulated OCT4 was unable to form colonies on soft agar (Fig. 2). Cell lineage markers were measured to assess the effect of OCT4 RNAi on BE (2)-C differentiation. Compared to cells infected with normal control 
A

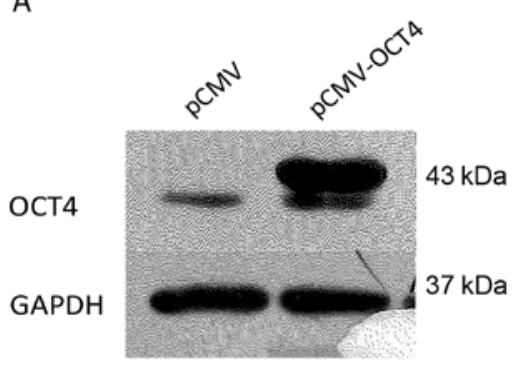

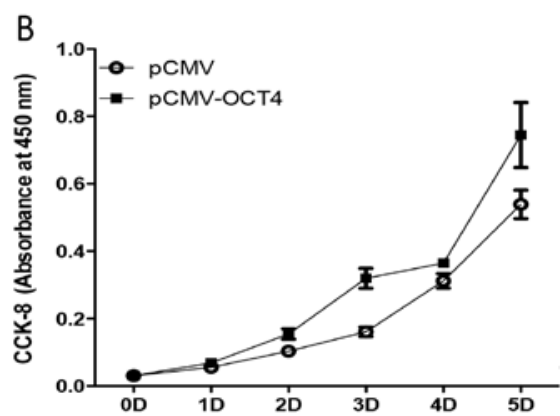

Figure 4. Upregulation of OCT4 in BE (2)-C cells. (A) Western blot analysis of OCT4 upregulation in BE (2)-c cells by transfection. GAPDH levels are shown as control. (B) OCT4 upregulation promoted proliferation of BE (2)-C cells. BE (2)-C cells transfected with OCT4 overexpression vector (pCMV-Oct4) or empty vector (pCMV). Cell growth rates were measured by Cell Counting Kit-8 (CCK-8) assay. Data are expressed as the means \pm SD with each point performed in triplicate. Independent experiments were repeated three times.

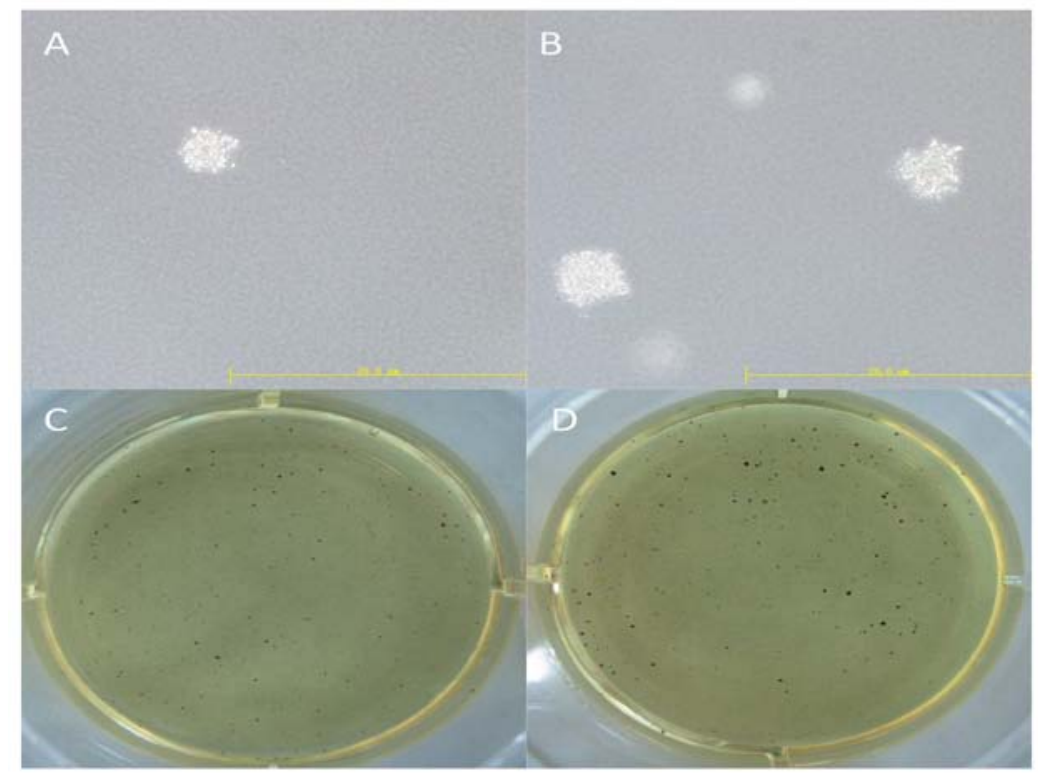

Figure 5. Measurement of self-renewal capacity of BE (2)-C cells with OCT4 upregulation using soft agar assay. (A) Micrographs of colonies of BE (2)-C/pCMV in soft agar 14 days after plating. (B) Colonies of BE (2)-C/pCMV-OCT4. (C and D) Colonies in one well corresponding to (A and B), respectively. Cells (1,000) were plated in each well. Viable colonies on soft agar were stained with $5 \mathrm{mg} / \mathrm{ml}$ MTT 14 days after plating. Scale bar, $20 \mu \mathrm{m}$.

vector by western blot analysis, BE (2)-C/OCT4 si cells showed an apparent decrease in the expression of N-type cell markers, peripherin and NF68, which was accompanied by an increase in the expression of the S-type cell markers, vimentin and S100 (Fig. 3C-E). The corresponding N- or S-type marker changes were also confirmed by quantitative RT-PCR analysis, which showed similar upregulation of vimentin and S100 and downregulation of peripherin and NF68 (Fig. 3F). Therefore, these results suggest that downregulation of OCT4 in BE (2)-C cells promotes cell differentiation into S-type cells.

Upregulation of OCT4 in BE (2)-C cells promotes proliferation without apparent differentiation. The lentiviral mediated OCT4 overexpression in BE (2)-C cells is shown by western blotting in Fig. 4A. OCT4-overexpressing BE (2)-C cells grew faster (Figure 4B) and colony formation of cells with upregulated OCT4 increased $6.5 \pm 2.2 \%$ using the soft agar assay (Fig. 5). However, protein levels of lineage differentiation marker of N-type (peripherin, NF68) and S-type (vimentin,
S100) remained relatively unaltered in the OCT4 upregulated cells as in the control cells (Fig. 6).

Reduced OCT4 expression accompanied by BrdUrd induces differentiation. RA and BrdUrd have been proved to induce differentiation of BE (2)-C into N-type and S-type cells, respectively, in vitro $(5,21)$. Following treatment of BrdUrd, in addition to the expected S-type morphological alteration, cells showed a progressive reduction of OCT4 expression levels in both protein and RNA levels, and there was no related change in induced $\mathrm{N}$-type cells (Fig. 7).

\section{Discussion}

Cancer stem cells possess the ability of high proliferation, selfrenewal and colony formation, and they finally cause cancer; they are similar to normal stem cells. Previous studies have isolated tumor-initiating cells with stem cell biological characteristics using various technologies in neuroblastoma (NB) $(4,21-24)$. 
A

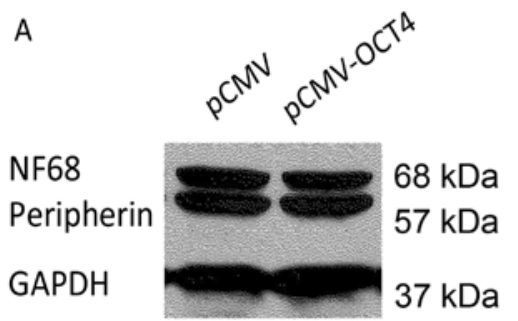

B

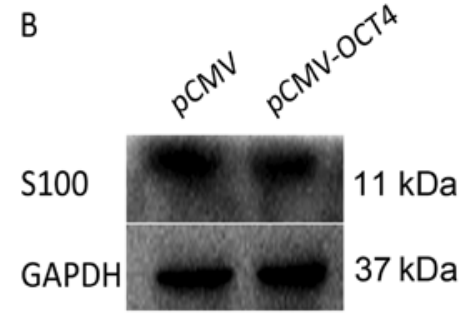

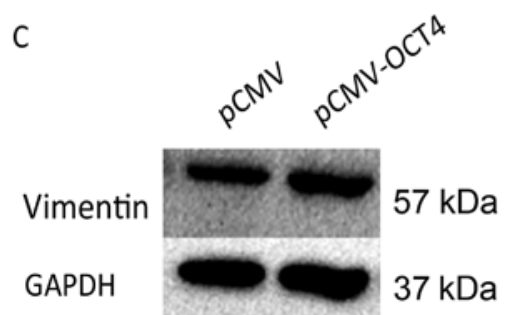

Figure 6. Western blot analysis of lineage differentiation markers of BE (2)-C/pCMV and BE (2)-C/pCMV-OCT4. (A) N-type markers of NF68 and peripherin. (B) S-type marker S100. (C) S-type marker vimentin. GAPDH levels are shown as control. All results were from cells transfected after 14 days.
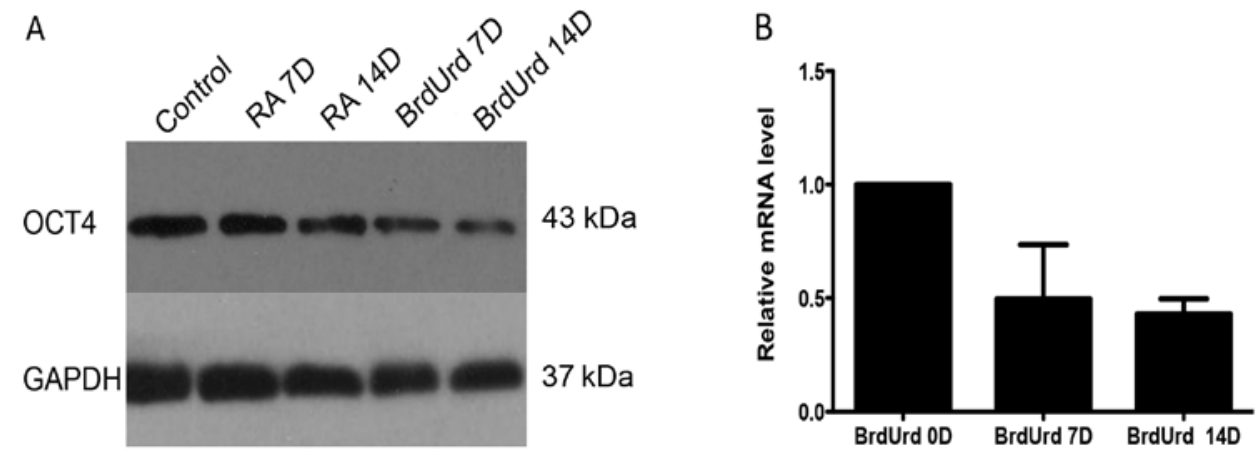

Figure 7. Western blot and quantitative RT-PCR analysis of OCT4 expression levels in BE (2)-C cells during the course of RA and BrdUrd treatment. (A) Compared with control BE (2)-C cells, cells treated with BrdUrd for 7 and 14 days displayed decreased expression levels of OCT4, while cells treated with RA had no significant reduction. GAPDH levels are shown as control. (B) Quantitative RT-PCR confirmed OCT4 expression decrease in cells treated with BrdUrd in RNA level. The mean expression in BE (2)-C cells was set at 1 . Data shown are the means \pm SD of three independent experiments.

Among them, the method of continuous NB cell culture in vitro identified three morphologically and biochemically phenotypic variants, a neuroblastic subunit (N-type), a flattened, substrate adherent subunit (S-type) representing Schwann/glial cells, and a third type characterized between $\mathrm{N}$ - and S-type, which is recognized as Intermediate type (I-type). Based on the following findings, I-type cells most closely resemble a population of malignant neural crest stem cells; first, I-type cells express markers of both $\mathrm{N}$ - and S-type cells and the specific stem cell proteins CD133 and c-kit; second, I-type cells differentiate to N- or S-type derivatives spontaneously or by specific agents; and third, I-type cells exhibit the greatest malignant potential in immune-deficient mice and higher clonogenic activity in soft agar than the other two types. Finally, presence of a larger number of I-type cells in the tumor has been reported to correlate with a poorer prognosis (6).

OCT4 has been used as a cell marker in NB to identify progenitor cells (12-17). Our study showed that the expression levels of OCT4 strongly correlated with the stemness of NB I-type cells BE (2)-C. High expression of OCT4 increases grades and correlates with tumor progression and malignancy in glioma, a nervous system malignant tumor (8). With the overexpression of OCT4, BE (2)-C cells acquired increased proliferation rate and formed relatively more and larger sphere colonies on soft agar, indicating that OCT4 also played a role in increasing malignancy in BE (2)-C. This is consistent with a previous study that indicates BE (2)-C spheres grown in serum-free non-adherent culture show increased expression of OCT4 (16). In addition, we showed that with the downregulation of OCT4, BE (2)-C grew slowly compared with control, and was unable to form colonies on soft agar. Soft agar is used as semisolid support media to prevent the migration of cells, which also leads to the formation of spatially distinct colonies. Anchorage-independent growth ability is a property of stem cells and formation of new colonies suggest self-renewal (21). This proved that OCT4 was essential in maintaining the clonogenic self-renewal capacity of BE (2)-C. Inability of growth in soft agar may result from defects in the regulation of proliferation or differentiation.

Since I-type NB cells can spontaneously differentiate into $\mathrm{N}$ - or S-type cell in vitro, we investigated the role of OCT4 in the regulation of $\mathrm{BE}$ (2)-C cell differentiation. Peripherin is expressed by neural crest-derived peripheral neurons and neuro- 
filament 68 (NF68) is an N-type specific marker protein (5), while vimentin is an intermediate filament highly expressed in S-type cells and S100 is a common marker for Schwann/glial cells (21). BE (2)-C cells with OCT4 downregulation became more flattened, substrate-adherent, which is a morphological characteristic of Schwann cells. In reduced expression of OCT4 cells, vimentin and S100 had a higher expression in both RNA and protein levels, while peripherin and NF68 expressed lower levels. Collectively, these results demonstrated that downregulation of OCT4 prompt BE (2)-C cells to exit from the I-type cell state and to differentiate into the Schwann/glial type. S-type non-neuronal cells are non-malignant and do not form tumors in athymic mice compared to I-type or N-type cells (5). OCT4 has been implicated in a variety of cellular functions including maintaining the pluripotent phenotype of embryonic stem cells (ESCs) and directing the differentiation of ESCs to particular cell lineages (25). It has also been used as a cancer stem cell marker and has the function to maintain cancer stem cell undifferentiation and malignancy in some types of cancer $(19,26,27)$. Hence, OCT4 is required to maintain the undifferentiating I-type state and a loss of OCT4 in NB cells might cause a differentiation into more benign S-types. This may also partially explain the inability of soft agar colony formation and further prove that OCT4 may enhance the malignancy and stem cell characteristics of NB cells.

In vivo, I-type cells originate from neural crest stem cells and represent a progenitor of $\mathrm{N}$ - and S-type cells $(5,6)$. In vitro, I-type $\mathrm{NB}$ cells were induced into $\mathrm{N}$-type cells by treating with retinoic acid (RA), whereas they were induced into S-type cells using BrdUrd (4,21). Our study revealed that BrdUrd induced differentiation of BE (2)-C into S-type accompanied with decreased expression of OCT4, whereas the RA-induced $\mathrm{N}$-type cells did not show apparent changes of OCT4 expression. Together with the observation of the BE (2)-C S-type differentiation with OCT4 downregulation, it is further suggested that OCT4 plays a role in regulating the BE (2)-C differentiation via the Schwann/glial pathway. Downregulation of OCT4 may cause BE (2)-C differentiation into S-type cells and vice versa.

Our study has some limitations. The mechanism of how OCT4 regulates the differentiation of BE (2)-C has not been investigated in this study and this experiment was only performed in vitro, thus it remains to be confirmed in vivo.

In summary, our study is consistent with the hypothesis that I-type NB cells are malignant neural crest stem cells. OCT4 may enhance the stem characteristics and be required to maintain the undifferentiated state of NB I-type cells by regulating the Schwann/glial pathway of BE (2)-C cells. Our study may provide a target for the treatment of NB.

\section{Acknowledgements}

This research was supported by the National Natural Science Foundation of China, grant no. 30801198.

\section{References}

1. Brodeur GM: Neuroblastoma: biological insights into a clinical enigma. Nat Rev Cancer 3: 203-216, 2003.

2. Maris JM, Hogarty MD, Bagatell R and Cohn SL: Neuroblastoma. Lancet 369: 2106-2120, 2007.
3. Shimada H, Umehara S, Monobe Y, et al: International neuroblastoma pathology classification for prognostic evaluation of patients with peripheral neuroblastic tumors: a report from the Children's Cancer Group. Cancer 92: 2451-2461, 2001

4. Ross RA, Spengler BA, Domenech C, Porubcin M, Rettig WJ and Biedler JL: Human neuroblastoma I-type cells are malignant neural crest stem cells. Cell Growth Differ 6: 449-456, 1995.

5. Walton JD, Kattan DR, Thomas SK, et al: Characteristics of stem cells from human neuroblastoma cell lines and in tumors. Neoplasia 6: 838-845, 2004.

6. Ross RA and Spengler BA: Human neuroblastoma stem cells. Semin Cancer Biol 17: 241-247, 2007.

7. Babaie Y,Herwig R, Greber B, et al: Analysis of Oct4-dependent transcriptional networks regulating self-renewal and pluripotency in human embryonic stem cells. Stem Cells 25: 500-510, 2007.

8. Du Z, Jia D, Liu S, et al: Oct4 is expressed in human gliomas and promotes colony formation in glioma cells. Glia 57: 724-733, 2009.

9. Chiou SH, Wang ML, Chou YT, et al: Coexpression of Oct4 and Nanog enhances malignancy in lung adenocarcinoma by inducing cancer stem cell-like properties and epithelial-mesenchymal transdifferentiation. Cancer Res 70: 10433-10444, 2010.

10. Ikushima $\mathrm{H}$, Todo $\mathrm{T}$, Ino $\mathrm{Y}$, et al: Glioma-initiating cells retain their tumorigenicity through integration of the Sox axis and Oct4 protein. J Biol Chem 286: 41434-41441, 2011.

11. Liu CG, Lu Y, Wang BB, et al: Clinical implications of stem cell gene Oct-4 expression in breast cancer. Ann Surg 253: 1165-1171, 2011.

12. Das B, Tsuchida R, Malkin D, Koren G, Baruchel S and Yeger H: Hypoxia enhances tumor stemness by increasing the invasive and tumorigenic side population fraction. Stem Cells 26: 1818-1830, 2008.

13. Melone MA, Giuliano M, Squillaro T, et al: Genes involved in regulation of stem cell properties: studies on their expression in a small cohort of neuroblastoma patients. Cancer Biol Ther 8: 1300-1306, 2009.

14. Pietras A, Hansford LM, Johnsson AS, et al: HIF-2alpha maintains an undifferentiated state in neural crest-like human neuroblastoma tumor-initiating cells. Proc Natl Acad Sci USA 106: 16805-16810, 2009

15. Newton TC, Wolcott K and Roberts SS: Comparison of the side populations in pretreatment and postrelapse neuroblastoma cell lines. Transl Oncol 3: 246-251, 2010.

16. Nishimura N, Hartomo TB, Pham TV, et al: Epigallocatechin gallate inhibits sphere formation of neuroblastoma $\mathrm{BE}(2)-\mathrm{C}$ cells. Environ Health Prev Med 17: 246-251, 2012.

17. Pezzolo A, Parodi F, Marimpietri D, et al: Oct-4(+)/Tenascin $\mathrm{C}(+)$ neuroblastoma cells serve as progenitors of tumor-derived endothelial cells. Cell Res 21: 1470-1486, 2011.

18. Lois C, Hong EJ, Pease S, Brown EJ and Baltimore D: Germline transmission and tissue-specific expression of transgenes delivered by lentiviral vectors. Science 295: 868-872, 2002.

19. Chen YC, Hsu HS, Chen YW, et al: Oct-4 expression maintained cancer stem-like properties in lung cancer-derived CD133positive cells. PLoS One 3: e2637, 2008.

20. Kutner RH, Zhang XY and Reiser J: Production, concentration and titration of pseudotyped HIV-1-based lentiviral vectors. Nat Protoc 4: 495-505, 2009.

21. Cui H, Ma J, Ding J, Li T, Alam G and Ding HF: Bmi-1 regulates the differentiation and clonogenic self-renewal of I-type neuroblastoma cells in a concentration-dependent manner. J Biol Chem 281: 34696-34704, 2006.

22. Marzi I, D'Amico M, Biagiotti T, et al: Purging of the neuroblastoma stem cell compartment and tumor regression on exposure to hypoxia or cytotoxic treatment. Cancer Res 67: 2402-2407, 2007.

23. Mahller YY, Williams JP, Baird WH, et al: Neuroblastoma cell lines contain pluripotent tumor initiating cells that are susceptible to a targeted oncolytic virus. PLoS One 4: e4235, 2009.

24. Coulon A, Flahaut M, Muhlethaler-Mottet A, et al: Functional sphere profiling reveals the complexity of neuroblastoma tumorinitiating cell model. Neoplasia 13: 991-1004, 2011.

25. Niwa H: How is pluripotency determined and maintained? Development 134: 635-646, 2007.

26. Chiou SH, Yu CC, Huang CY, et al: Positive correlations of Oct-4 and Nanog in oral cancer stem-like cells and high-grade oral squamous cell carcinoma. Clin Cancer Res 14: 4085-4095, 2008.

27. Kim R-J and Nam J-S: OCT4 Expression enhances features of cancer stem cells in a mouse model of breast cancer. Lab Anim Res 27: 147-152, 2011. 